\title{
Should FDG PET/CT be used for the initial staging of breast cancer?
}

\author{
David Groheux • Elif Hindié • Domenico Rubello • \\ Marc Espié • Georges Baillet • Sylvie Giacchetti • \\ Jean-Louis Misset • Jean-Luc Moretti
}

Published online: 16 May 2009

(C) Springer-Verlag 2009

Previous studies have pointed to the suboptimal sensitivity of ${ }^{18} \mathrm{~F}$-FDG PET in assessing axillary lymph node status in breast cancer patients [1,2]. In this issue of The European Journal of Nuclear Medicine and Molecular Imaging, Heusner and colleagues from the University Hospital of Essen bring further evidence to this statement. The study shows that contrast-enhanced full-dose FDG PET/CT is also not able to replace the sentinel lymph node biopsy technique (SLNB) or axillary lymph node dissection (ALND) for axillary staging [3]. The authors retrospectively examined the data of 61 consecutive women with histopathologically confirmed breast cancer who had received an i.v. contrastenhanced whole-body FDG PET/CT. Twenty-four patients (39\%) had preoperative clinical stage T1 [4], while the others had larger tumours. In all patients, the ipsilateral

This Editorial Commentary refers to the article http://dx.doi.org/ 10.1007/s00259-009-1145-6

\footnotetext{
D. Groheux $\cdot$ E. Hindié $(\bowtie) \cdot$ J.-L. Moretti

Department of Nuclear Medicine, Hôpital Saint-Louis,

Assistance publique-Hopitaux de Paris,

1 avenue Claude Vellefaux,

75475 Paris Cedex 10, France

e-mail: elif.hindie@sls.aphp.fr

D. Rubello $(\bowtie)$

Department of Nuclear Medicine, PET Centre,

'Santa Maria della Misericordia' Hospital,

Rovigo, Italy

e-mail: domenico.rubello@libero.it
}

M. Espié · S. Giacchetti · J.-L. Misset

Department of Medical Oncology and Breast Diseases Unit,

Hôpital Saint-Louis,

Paris, France

G. Baillet

Department of Nuclear Medicine and Oncology, PZQ Hospital, Fort-de-France, France axilla was assessed for lymph node metastases either with SLNB, ALND or both.

Of the 61 patients, 24 had axillary lymph node metastases. The sensitivity of FDG PET/CT on a patient basis was $58 \%$ [3]. The ten axillary fossae which were falsely negative on FDG PET/CT contained 1.8 lymph node metastases on average (range: 1-4). In one case, a $2.4 \mathrm{~cm}$ invaded lymph node immediately adjacent to the primary tumour was overlooked by FDG PET/CT because of mistaking it as a part of the primary tumour. In the other missed cases, the size of the metastatic deposits ranged from 0.8 to $6 \mathrm{~mm}$. FDG findings in the axillary fossae came out to be false-positive in three patients. The positive predictive value was $82 \%(14 / 17)$. The authors conclude that FDG PET/CT cannot replace invasive approaches for axillary staging in breast cancer patients but may be helpful as a pre-test for the triage to SLNB versus ALND.

\section{Can FDG PET/CT influence the decision on SLNB versus ALND?}

Today, SLNB has widely been accepted in small primary tumours as a less invasive alternative to ALND [5]. However, there is still debate about its use in tumours larger than 2 $3 \mathrm{~cm}$, and many authors prefer maintaining routine ALND. Reluctance of some authors to use SLNB in tumours larger than $2-3 \mathrm{~cm}$ is linked to several reasons: The false-negative rate of SLNB (usually close to $5-10 \%$ ) may be judged too high to be applied to this population with high prevalence of axillary lymph node metastases ( $\geq 40 \%)$. Also, if pathology results are not known immediately, a substantial number of patients with positive SLNB would be exposed to a second surgical procedure (11 patients in the study of Heusner et al. underwent SLNB and subsequent ALND) [3]. 
Today, patients with $\mathrm{T} 1(\leq 2 \mathrm{~cm})$ tumours represent the majority of newly diagnosed breast cancer patients, given improved screening in Western countries. FDG PET/CT has probably no role at initial staging in these patients. Sensitivity in detecting axillary involvement is too low and cannot be used as a substitute to SLNB. The probability of finding distant metastases is also low.

The situation is different when considering the use of SLNB in patients with larger tumours (especially so beyond $3 \mathrm{~cm}$ ). In these patients, it seems wise to try to exclude macrometastases by an imaging technique, such as ultrasound coupled with fine-needle biopsy of a suspicious node, or FDG PET/CT, before applying the SLNB procedure [5]. By identifying $50 \%$ or more of cases of clinically occult lymph node disease in the axilla, FDG $\mathrm{PET} / \mathrm{CT}$ will reduce the risk linked to false-negative SLNB. It is important to point out that in some cases a falsenegative SLNB can be due to massive invasion of a sentinel node that has lost its functional capacity of phagocytosis. Because the positive predictive value is high $(82 \%$ in the study of Heusner et al.), patients with positive FDG PET/ $\mathrm{CT}$ in the axilla will receive ALND, thus avoiding the risk of a two-step surgical procedure.

In many centres, patients with large breast tumours are treated by primary chemotherapy before breast surgery. There is large debate on whether SLNB is accurate enough after neoadjuvant chemotherapy or if it should be performed before starting chemotherapy [5]. FDG PET/CT might be helpful in this regard. When findings at the axilla are unremarkable on FDG PET/CT before chemotherapy, this would increase the level of confidence, making a postchemotherapy SLNB procedure an acceptable choice.

While FDG PET/CT might serve in expanding the use of SLNB, there are many other justifications for the use of FDG PET/CT at initial staging of patients with large breast tumours. Several studies have documented the value of ${ }^{18} \mathrm{~F}$ FDG PET in locally advanced and inflammatory breast cancer $[6,7]$, but also in populations comprising clinical stage II patients $[8,9]$.

We hereafter discuss the different roles that PET/CT may play in initial assessment.

\section{Primary tumour assessment}

In the study of Heusner et al., the primary tumour was FDG PET positive in $87 \%$ of cases [3]. However, $39 \%$ of patients had clinical stage T1. Given the limited spatial resolution, FDG PET can miss small lesions, and this is especially true for low-grade tumours and lobular carcinoma [10]. In a recent study, among 32 invasive tumours $>2 \mathrm{~cm}, 31$ (97\%) were well visualized with FDG PET [8]. PET might, however, miss additional small foci of breast cancer. The $\mathrm{T}$ stage of large tumours and multifocality are usually assessed with MRI.

In practice, the main contribution of PET in local tumour assessment consists in measuring FDG uptake using the standardized uptake value (SUV), which is useful to evaluate early response to preoperative primary chemotherapy $[11,12]$. Assessing early response with PET could be useful to avoid maintaining an ineffective chemotherapy and to decide optimal timing for surgery.

\section{Axillary and extra-axillary lymph node staging}

Heusner and colleagues do not provide us with a full description of the anatomical distribution of involved nodes. Depicting lymph node involvement in levels or basins other than those addressed by routine ALND may have major impact on treatment strategies. Many studies suggest that FDG PET performs better than conventional imaging in depicting involvement in high-level axillary (Berg level III) as well as in supraclavicular and internal mammary lymph nodes $[8,9,13-15]$. PET/CT is particularly appealing compared to PET alone as it provides the precise location of involved nodes [7-9]. Also, falsepositives resulting from muscular and brown fat uptake are avoided. In a prospective study of 60 breast tumours larger than $3 \mathrm{~cm}$, PET/CT revealed unsuspected infraclavicular node involvement (N3a) in two patients, supraclavicular node involvement $(\mathrm{N} 3 \mathrm{c})$ in one patient and contralateral axillary involvement (M1) in one patient [9]. Differentiating level III axillary lymph nodes (infraclavicular lymph nodes located superomedially to the pectoralis minor muscle) is important since axillary clearance usually addresses Berg level I and II nodes only. Surgical studies have demonstrated that level III node involvement (N3a) results in a poorer prognosis [16]; a modified surgical approach is then useful [8].

Detection of extra-axillary involvement (supraclavicular and/or internal mammary lymph nodes) is also very helpful in delineating radiotherapy target zone or schedule surgery. Patients with supraclavicular lymph nodes $(\mathrm{N} 3 \mathrm{c})$ can receive more intensive treatment combining induction chemotherapy, surgery, postsurgical chemotherapy and irradiation, which improves the disease-free survival and overall survival [17]. Again, visualization of an internal mammary hot node on initial PET/CT can lead to the decision on surgery and/or radiotherapy [8].

\section{Assessing distant metastases}

The findings of metastasis on the initial work-up radically changes treatment approaches. FDG PET [14, 18, 19] and 
especially FDG PET/CT [7-9] perform better than classic modalities to demonstrate occult metastases. In a retrospective study on 41 patients with inflammatory breast cancer, PET/CT demonstrated distant metastases in 20. In seven of these patients, metastatic involvement was initially missed by conventional imaging [7].

Two prospective studies have evaluated PET/CT in the initial work-up of less advanced breast cancer $[8,9]$. Our study included 39 patients with stage II or III breast cancer after conventional work-up [8]. PET/CT revealed occult metastases in four patients. These were bone metastases in three patients and pleural involvement in one patient [8]. Fuster et al. studied 60 consecutive patients with breast cancer stage IIb or higher [9]. PET/CT sensitivity and specificity in detecting distant metastasis were 100 and $98 \%$, respectively, versus 60 and $83 \%$ for conventional work-up (contrast-enhanced chest CT, liver ultrasonography, ${ }^{99 \mathrm{~m}} \mathrm{Tc}-\mathrm{HDP}$ bone scan). Metastases missed by conventional work-up were visualized in eight patients. The sites of involvement were bone (six cases), and/or lung (two cases) and/or liver (two cases).

In the two above-mentioned studies, bone was the most frequent site of occult distant metastasis documented with PET/CT. It is well acknowledged that FDG PET performs better than bone scan for lytic metastases and for early intra-medullary involvement, but has lower sensitivity in cases of pure osteoblastic lesions [20].

$\mathrm{PET} / \mathrm{CT}$ is also highly sensitive in detecting pleural, mediastinal, abdominal and pelvic metastases. In the study by Fuster et al., PET/CT diagnosed both patients with liver metastases, whereas liver ultrasound detected only one of the two cases [9]. PET performs well to assess lung nodules larger than $1 \mathrm{~cm}$, but it lacks sensitivity in smaller lesions, due to partial volume effect and respiratory motion. Careful reading of the CT images may depict small pulmonary nodules that do not present as hot spots on PET. However, CT obtained under free breathing conditions is still less sensitive than classic (breath-holding) CT. The main limitation of PET is its low sensitivity for brain metastases.

There remain some uncertainties about which clinical stages of breast cancer require a PET study. Routine use of PET may expose subjects to the drawback of false-positive findings. The ratio of false-positive to true-positive increases with decreasing stage. Large studies are therefore necessary to better assess the specific role of PET/CT in stage II patients, and in each of the subgroups IIa and IIb.

In conclusion, based on available literature, PET/CT appears to perform better than conventional modalities in the initial work-up of breast cancer patients. PET/CT allows diagnosis of infraclavicular, supraclavicular and internal mammary node involvement and can depict occult distant metastases. Preliminary findings from Heusner and colleagues suggest that the information provided by FDG PET/
CT might be useful in extending the indication of SLNB to patients with tumours larger than $2-3 \mathrm{~cm} \mathrm{[3].} \mathrm{The} \mathrm{role} \mathrm{of}$ PET in assessing response to neoadjuvant chemotherapy is also growing, and a baseline PET is necessary for that purpose. PET/CT at initial staging could thus become an unavoidable modality to answer a large set of clinical situations. But the exact clinical stage (III, IIb, IIa) for which PET/CT could be systematically performed with a favourable balanced cost-effectiveness is uncertain. A large multi-institutional trial should be encouraged to clarify this point.

\section{References}

1. Wahl RL, Siegel BA, Coleman RE, Gatsonis CG, PET Study Group. Prospective multicenter study of axillary nodal staging by positron emission tomography in breast cancer: a report of the staging breast cancer with PET Study Group. J Clin Oncol 2004;22:277-85. doi:10.1200/JCO.2004.04.148.

2. Veronesi U, De Cicco C, Galimberti VE, Fernandez JR, Rotmensz $\mathrm{N}$, Viale $\mathrm{G}$, et al. A comparative study on the value of FDG-PET and sentinel node biopsy to identify occult axillary metastases. Ann Oncol 2007;18:473-8. doi:10.1093/annonc/mdl425.

3. Heusner TA, Kuemmel S, Hahn S, Koeninger A, Otterbach F, Hamami ME, et al. Diagnostic value of full-dose FDG-PET/CT for axillary lymph node staging in breast cancer patients. Eur J Nucl Med Mol Imaging. 2009; In press.

4. Singletary SE, Allred C, Ashley P, Bassett LW, Berry D, Bland KI, et al. Revision of the American Joint Committee on Cancer staging system for breast cancer. J Clin Oncol 2002;20:3628-36. doi:10.1200/JCO.2002.02.026.

5. Hindié E, Groheux D, Espié M, Bourstyn E, Toubert ME, Sarandi F, et al. Sentinel node biopsy in breast cancer [review]. Bull Cancer 2009; In press.

6. van der Hoeven JJ, Krak NC, Hoekstra OS, Comans EF, Boom $\mathrm{RP}$, van Geldere $\mathrm{D}$, et al. 18F-2-fluoro-2-deoxy-D-glucose positron emission tomography in staging of locally advanced breast cancer. J Clin Oncol 2004;22:1253-9. doi:10.1200/ JCO.2004.07.058.

7. Carkaci S, Macapinlac HA, Cristofanilli M, Mawlawi O, Rohren E, Gonzalez Angulo AM, et al. Retrospective study of 18F-FDG PET/ $\mathrm{CT}$ in the diagnosis of inflammatory breast cancer: preliminary data. J Nucl Med 2009;50:231-8. doi:10.2967/jnumed.108.056010.

8. Groheux D, Moretti JL, Baillet G, Espie M, Giacchetti S, Hindie E, et al. Effect of (18) F-FDG PET/CT imaging in patients with clinical stage II and III breast cancer. Int J Radiat Oncol Biol Phys 2008;71:695-704. doi:10.1016/j.ijrobp. 2008.02.056.

9. Fuster D, Duch J, Paredes P, Velasco M, Muñoz M, Santamaría $\mathrm{G}$, et al. Preoperative staging of large primary breast cancer with [18F]fluorodeoxyglucose positron emission tomography/ computed tomography compared with conventional imaging procedures. J Clin Oncol 2008;26:4746-51. doi:10.1200/JCO. 2008.17.1496.

10. Gil-Rendo A, Martínez-Regueira F, Zornoza G, García-Velloso MJ, Beorlegui C, Rodriguez-Spiteri N. Association between [18F] fluorodeoxyglucose uptake and prognostic parameters in breast cancer. Br J Surg 2009;96:166-70. doi:10.1002/bjs.6459.

11. Berriolo-Riedinger A, Touzery C, Riedinger JM, Toubeau M, Coudert B, Arnould L, et al. [18F]FDG-PET predicts complete pathological response of breast cancer to neoadjuvant chemother- 
apy. Eur J Nucl Med Mol Imaging 2007;34:1915-24. doi:10.1007/ s00259-007-0459-5.

12. Schwarz-Dose J, Untch M, Tiling R, Sassen S, Mahner S, Kahlert S, et al. Monitoring primary systemic therapy of large and locally advanced breast cancer by using sequential positron emission tomography imaging with $[18 \mathrm{~F}]$ fluorodeoxyglucose. J Clin Oncol 2009;27:535-41. doi:10.1200/JCO.2008.17.2650.

13. Danforth DN Jr, Aloj L, Carrasquillo JA, Bacharach SL, Chow C, Zujewski J, et al. The role of $18 \mathrm{~F}-\mathrm{FDG}-\mathrm{PET}$ in the local/regional evaluation of women with breast cancer. Breast Cancer Res Treat 2002;75:135-46. doi:10.1023/A:1019664126220.

14. Cermik TF, Mavi A, Basu S, Alavi A. Impact of FDG PET on the preoperative staging of newly diagnosed breast cancer. Eur J Nucl Med Mol Imaging 2008;35:475-83. doi:10.1007/s00259-007-0580-5.

15. Bellon JR, Livingston RB, Eubank WB, Gralow JR, Ellis GK, Dunnwald LK, et al. Evaluation of the internal mammary lymph nodes by FDG-PET in locally advanced breast cancer (LABC). Am J Clin Oncol 2004;27:407-10. doi:10.1097/01.coc.000012 8869.19357.9b.

16. Kuru B, Camlibel M, Dinc S, Gulcelik MA, Alagol H. Prognostic significance of axillary node and infraclavicular lymph node status after mastectomy. Eur J Surg Oncol 2003;29:839-44. doi:10.1016/ j.ejso.2003.08.003

17. Brito RA, Valero V, Buzdar AU, Booser DJ, Ames F, Strom E, et al. Long-term results of combined-modality therapy for locally advanced breast cancer with ipsilateral supraclavicular metastases: The University of Texas M.D. Anderson Cancer Center experience. J Clin Oncol 2001;19:628-33.

18. Port ER, Yeung H, Gonen M, Liberman L, Caravelli J, Borgen P, et al. 18F-2-fluoro-2-deoxy-D-glucose positron emission tomography scanning affects surgical management in selected patients with high-risk, operable breast carcinoma. Ann Surg Oncol 2006;13:677-84. doi:10.1245/ASO.2006.03.035.

19. Mahner S, Schirrmacher S, Brenner W, Jenicke L, Habermann CR, Avril N, et al. Comparison between positron emission tomography using 2-[fluorine-18]fluoro-2-deoxy-D-glucose, conventional imaging and computed tomography for staging of breast cancer. Ann Oncol 2008;19:1249-54. doi:10.1093/annonc/mdn057.

20. Nakai T, Okuyama C, Kubota T, Yamada K, Ushijima Y, Taniike K, et al. Pitfalls of FDG-PET for the diagnosis of osteoblastic bone metastases in patients with breast cancer. Eur J Nucl Med Mol Imaging 2005;32:1253-8. doi:10.1007/s00259-005-1842-8. 